\title{
AUGUSTUS
}

\section{ASPECTOS DE TELEPROTEÇÃO PARA LINHAS DE TRANSMISSÃO: REVISÃO BIBLIOGRÁFICA}

André da Costa Pinho

Doutor em Engenharia de Sistemas e Computação pela Universidade Federal do

Rio de Janeiro (UFRJ), Rio de Janeiro, RJ, Brasil

acpinho@id.uff.br

Angelo Cesar Colombini Doutor em Física Computacional pela Universidade de São Paulo (USP), São Paulo, SP, Brasil accolombini@id.uff.br

Mayara Helena Moreira Nogueira dos Santos Graduando em Engenharia Elétrica na Universidade Federal Fluminense (UFF), Rio de Janeiro, RJ, Brasil mayarahelena@id.uff.br

Matheus Felipe Ayello Leite Graduando em Engenharia Elétrica na Universidade Federal Fluminense (UFF), Rio de Janeiro, RJ, Brasil matheus_felipe@id.uff.br

\section{RESUMO}

Os sistemas de teleproteção exercem papel fundamental para garantir a confiabilidade da transmissão da energia elétrica, permitindo a previsibilidade de atendimento às demandas nacionais. Esses sistemas configuram-se como arranjos compostos por esquemas de proteção associados a subsistemas de telecomunicações. A literatura técnica e científica, em geral, traz esses conceitos bastante segregados. Este trabalho tem por objetivo apresentar uma revisão bibliográfica do tema, detalhando aspectos técnicos sobre o sistema de transmissão, equipamentos, funções de proteção aplicáveis e sistemas de telecomunicações. $\mathrm{O}$ texto permite uma avaliação comparativa entre diversas tecnologias e o resultado final traduz-se em uma fonte de informações consolidada acerca do assunto, tornando-se uma importante referência para engenheiros e pesquisadores que venham a trabalhar em projetos relacionados a linhas de transmissão.

Palavras-chave: Proteção de sistemas de potência. Linhas de transmissão. Serviços de telecomunicações.

\section{TELEPROTECTION ASPECTS FOR TRANSMISSION LINES: BIBLIOGRAPHIC REVIEW}

\begin{abstract}
Teleprotection systems play a fundamental role in ensuring the electricity transmission reliability, allowing the predictability of meeting national demands. These systems are
\end{abstract}


configured as arrangements composed of protection schemes associated with telecommunications subsystems. Technical and scientific literature, in general, bring these concepts quite segregated. This paper aims to present a bibliographic review of this theme, detailing technical aspects about the transmission system, equipment, applicable protection functions and telecommunications systems. The text allows for a comparative assessment between different technologies and is a consolidated source of information on this subject, becoming an important reference for engineers and researchers who will work on projects related to transmission lines.

Keywords: Power system protection. Transmission lines. Telecommunication services.

Recebido: 01/04/2020. Aceito: 08/10/2020. 


\section{INTRODUÇÃO}

Atualmente a energia elétrica figura não só como um item estruturante da economia de um país, mas também como uma necessidade básica de toda população. Estas demandas impuseram o desenvolvimento e a implantação de sistemas complexos de transmissão e distribuição para o atendimento de consumidores. Sistemas desta natureza estão sujeitos a uma infinidade de eventos (intempéries, mau funcionamento de equipamentos etc.) que podem trazer prejuízos de ordem material e humana, tanto para as concessionárias quanto para os consumidores. Desta forma, mecanismos de proteção devem ser capazes de atuar prontamente para isolar, em uma menor região possível, o defeito/falha, de maneira a prover a segurança necessária e interromper o fornecimento de energia para o mínimo de consumidores. Ressalta-se que a referida atuação depende de inúmeros parâmetros da rede e que os dispositivos envolvidos podem estar a longas distâncias um dos outros.

Os sistemas de proteção devem atender de acordo com (CIGRÉ, 2001) aos requisitos de sensibilidade, velocidade, seletividade e confiabilidade para atuar de forma adequada na ocorrência de falhas. Tais requisitos estão descritos em (MAMEDE FILHO; MAMEDE, 2011).

Sistemas tradicionais de proteção de geradores, barras, transformadores ou outras unidades dentro de uma subestação (SE), normalmente atendem a alguns destes requisitos sem requerer o uso de sistemas de telecomunicações, no entanto, esses não podem ser aplicados diretamente ao contexto das Linhas de Transmissão (LTs), uma vez que a exigência dos requisitos de velocidade e seletividade são bem maiores. (CIGRÉ, 2001).

Neste contexto surge a demanda pela aplicação da teleproteção, ou seja, circuitos de proteção baseados no uso de sistemas de telecomunicações capazes de atender a robustos requisitos de velocidade de atuação e seletividade.

Este trabalho tem por objetivo revisitar os principais aspectos técnicos relacionados ao projeto de sistemas de teleproteção, tornando-se uma importante referência para profissionais que trabalhem ou venham a trabalhar na área de transmissão de energia elétrica. 


\section{METODOLOGIA}

Este artigo foi elaborado com base em uma pesquisa bibliográfica exploratória acerca dos principais conceitos e tecnologias relacionados à teleproteção. Os conceitos de proteção de sistemas de transmissão de energia elétrica praticamente não sofreram alterações sensíveis nas últimas décadas e, por isso, as referências exploradas basearam-se em livros e publicações técnicas e científicas de autores consagrados. No tocante à inserção de dispositivos eletrônicos inteligentes nas redes de energia e à evolução dos sistemas de telecomunicações, foram buscadas as publicações mais atuais que revelassem o estado da arte dessas tecnologias. Normas e publicações técnicas de diversos fabricantes enriqueceram o texto com inúmeros parâmetros e requisitos a serem considerados em projetos de teleproteção. A organização do texto permite comparar o desempenho de diferentes abordagens tecnológicas, constituindo-se em importante fonte de consulta para análise do tema.

\section{SISTEMAS ELÉTRICOS DE POTÊNCIA (SEP)}

Segundo Cigré (2001), os sistemas de energia elétrica podem ser divididos em três núcleos principais: unidades geradoras, sistemas de transmissão e sistemas de distribuição. Conectados ao sistema de geração, estão os sistemas de transmissão que são compostos pelas LT e pelas subestações (SEs). No Sistema Elétrico Brasileiro (SEB), as LTs operam com tensões entre $230 \mathrm{kV}$ e $765 \mathrm{kV}$ em corrente alternada e $500 \mathrm{kV}$ a $800 \mathrm{kV}$ em corrente contínua conforme Operador Nacional do Sistema Elétrico (ONS, 2011a). A transmissão que une a LT à subestação da rede principal possui tensão superior que 200 kV. Já a subtransmissão que conecta a LT às cargas opera com tensões entre 69 e 138 kV.

O sistema de distribuição é constituído pelo conjunto de linhas, alimentadores, ramais de serviços e estações abaixadoras, que se destinam a atender o consumidor final, operando com tensão de serviço entre 110/220 V e 35 kV.

O SEP deve atender aos desafios de expansão da capacidade de geração e transmissão de energia, de forma a suprir a demanda energética da sociedade. Para que isso seja possível, investimentos em infraestrutura são necessários, incluindo a construção de novos empreendimentos de geração, transmissão e distribuição. Para tal, são empregados 
diversos equipamentos, dos quais se destacam: geradores, transformadores, LT, chaves seccionadoras e disjuntores.

Dando foco à LT, que é o tema deste artigo, segundo Pinto (2014), sua função primária é transportar a energia elétrica com o mínimo de perdas, do centro de geração aos centros de carga, geralmente separados por distâncias elevadas. O Brasil possui um sistema de transmissão extenso, refletindo as dimensões continentais do país. Segundo o Ministério de Minas e Energia (2018) tem-se 144.324 km de linhas instaladas no Brasil, conforme informações constantes na Tabela 1.

Tabela 1 - Linhas de Transmissão de Energia Elétrica.

\begin{tabular}{|c|c|c|}
\hline Classe de Tensão $(\mathrm{kV})$ & Linhas de Transmissão Instaladas $(\mathrm{km})$ & \% Total \\
\hline 230 & 57.552 & 39,9 \\
\hline 345 & 10.319 & 7,2 \\
\hline 440 & 6.748 & 4,7 \\
\hline 500 & 49.606 & 34,4 \\
\hline $600(\mathrm{CC})$ & 12.816 & 8,9 \\
\hline 750 & 2.683 & 1,9 \\
\hline $800(\mathrm{CC})$ & 4.600 & 3,2 \\
\hline Total & 144.324 & 100 \\
\hline
\end{tabular}

Fonte: Ministério de Minas e Energia (2018, p. 14).

Uma das soluções existentes para transmissão de informações utilizando o meio óptico guiado é o sistema denominado OPGW (Optical Ground Wire) (ARAGÃO et al., 2016), que consiste em um cabo guarda aterrado com uma fibra óptica percorrendo seu eixo axial. É bastante utilizado em linhas de transmissão e distribuição, provendo, além de proteção elétrica, canais de comunicação de alta capacidade de transmissão de dados com elevada imunidade eletromagnética (DECHUN; ZHIHUI; JIE, 2016). Tem-se ainda a fibra de carbono como uma solução em estudo, conforme detalhado em (LESSA et al., 2016).

\subsection{Motivação para proteção dos equipamentos do SEP}

Os investimentos requeridos para a construção de um SEP, assim como os custos para reposição dos seus componentes, fazem com que existam diversos requisitos para a implantação de um sistema de proteção. Dentre outras funções, este sistema deve garantir a integridade dos equipamentos do SEP. 
Os dispositivos de proteção integrados ao SEP devem estar adequadamente posicionados, além de possuir ajustes que garantam a eficácia do sistema de proteção.

O custo dos equipamentos aplicados nas LTs e SEs é um fator crítico que influencia decisivamente no projeto de proteção. Pode-se citar, como exemplos, os custos extraídos do banco de dados da Aneel (referência junho de 2017) para um transformador de 100 MVA (500/345 kV), R\$1 13.704.227,69, e para um banco de capacitores 100 MVAr a $230 \mathrm{kV}, \mathrm{R} \$$ $17.509 .025,64$.

Além da questão econômica apresentada anteriormente, o sistema de proteção tem papel relevante na operação do sistema elétrico, pois têm como objetivo assegurar que, na ocorrência de um evento, seja possível desconectar a parte em falha do sistema elétrico interligado, facilitando a detecção e a resolução das causas da falha. (MAMEDE FILHO; MAMEDE, 2011).

\section{PROTEÇÃO}

Como apontado na introdução, os SEP demandam altos índices de confiabilidade em seu funcionamento. No entanto, estes sistemas possuem equipamentos sujeitos a falhas de origem externa e interna. A eventual indisponibilidade de trechos do sistema traz prejuízos tanto para a empresa responsável por sua manutenção e operação, quanto para o consumidor, que terá seu fornecimento de energia interrompido (KINDERMANN, 2005). Dessa forma, é desejável que os equipamentos sejam desconectados o mais rápido possível para se reduzir o tempo de exposição à falha.

Para o Sistema Interligado Nacional (SIN), o Operador Nacional do Sistema (ONS) define metodologias que devem ser utilizadas nos sistemas de proteção e teleproteção implementados pelos agentes de transmissão e geração. Segundo o ONS (2011b), estes sistemas são de responsabilidade dos agentes e são constituídos pelo conjunto de dispositivos "necessários e suficientes para a deteç̧ão e eliminação, de forma seletiva, de todos os tipos de faltas (...) e de outras condições anormais de operação".

Todo sistema de proteção deve atender a requisitos cujos detalhamentos estão apresentados em (FERRER; SCHEITZER, 2010).

Outro conceito importante em proteção é a coordenação dos dispositivos envolvidos. De acordo com Santos, Bernardes e Asada (2013), um relé não deve operar para 
faltas externas ao equipamento protegido e, portanto, devem ser levados em consideração os tempos de sensibilização e de processamento do comando, para garantir uma atuação coordenada.

Em IEEE (2013) são abordados dois critérios para avaliação dos sistemas de proteção: confiabilidade e segurança. Entendida como um critério de nível mais alto, a segurança mede a habilidade do sistema de proteção em operar somente nas faltas desejadas. Segundo Cigré (2001), este critério é atendido com alta seletividade e baixo tempo de resposta de modo a minimizar a probabilidade de desligamentos indesejados. Em um nível mais baixo, a confiabilidade é a habilidade de um sistema de proteção responder a todas as faltas internas. Conforme observado em Cigré (2001), nota-se um conflito entre estes dois critérios, que deve ser harmonizado em uma relação de compromisso que permita o projeto de um sistema de proteção confiável.

Como critério de confiabilidade para o SIN, o ONS exige (ONS, 2011b) que todo componente seja protegido localmente por dois sistemas de proteção independentes, chamados de proteção principal e alternada. A independência entre essas redundâncias impõe subsistemas de alimentação, instrumentação e telecomunicações individualizados.

Em Fortes, Ferreira e Zanghi (2015) foi apresentada uma pesquisa sobre diagnóstico de faltas em LTs e alguns conceitos importantes a serem destacados para sistemas de proteção são:

\subsection{Zonas de Proteção (ZP)}

Uma ZP é uma fronteira teórica estabelecida ao redor de cada equipamento do sistema para delimitar a área de atuação (interna ou externa) dos elementos de proteção. Os limites da zona são definidos através de disjuntores colocados na conexão entre dois equipamentos quaisquer.

Na Figura 1, cada zona está associada a um equipamento do SEP e há disjuntores responsáveis pelo seccionamento do equipamento do resto do sistema. Verifica-se a necessidade da coordenação adequada da proteção, visto que as zonas se sobrepõem. Essa sobreposição é intencional, pois os sistemas de proteção estão sujeitos a falhas e, por isso, as zonas sobrepostas atuam como uma proteção de retaguarda entre si. 
Figura 1 - Zonas de Proteção.

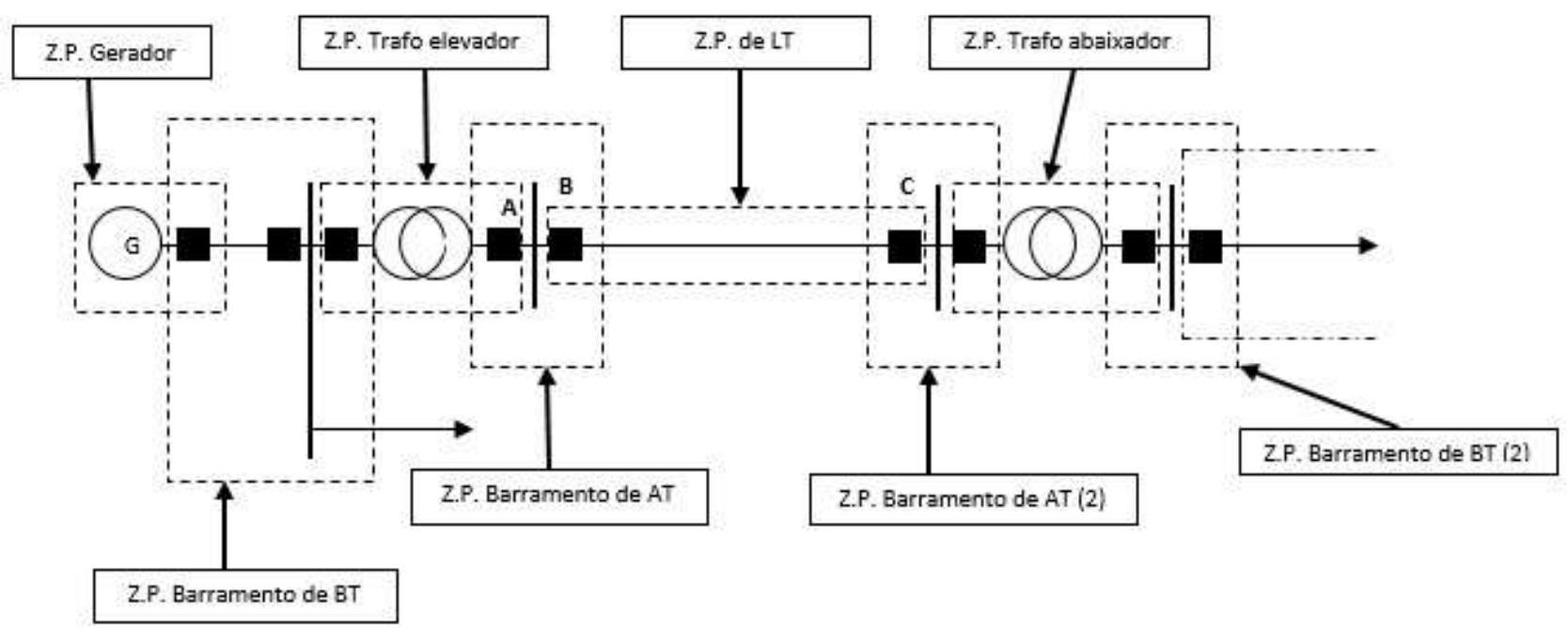

Fonte: Autor (2020).

De acordo com Mamede Filho e Mamede (2011), podem ser utilizadas múltiplas zonas de proteção, com abrangências ou alcances diferentes entre si. O sobrealcance estende a zona para além do equipamento protegido e o subalcance considera apenas falta no equipamento. Frequentemente são utilizadas as nomenclaturas zona 1 para subalcance e zona 2 para sobrealcance, mas vale ressaltar que isso pode variar. Há linhas que possuem um número superior de zonas definidas e essas zonas adicionais podem ser reversas (defasagem de $180^{\circ}$ entre corrente e tensão).

\subsection{Funções de Proteção}

As funções de proteção são os arranjos eletromecânicos que foram criados para detectar e isolar as faltas. As funções foram padronizadas na norma IEEE (2008), a partir da tabela ANSI, onde há uma relação direta entre a numeração da função e o relé a ela associada. As funções de proteção mais comuns aplicadas às LTs são: 50 - sobrecorrente instantânea; 51 - sobrecorrente temporizada; 21 - distância; 87L - diferencial de linha e 67 direcional de sobrecorrente.

Nos relés eletromecânicos cada função era desempenhada por um único relé de proteção. Com o advento dos relés digitais, surgiu a possibilidade de se implementar várias funções em um mesmo dispositivo. Esses dispositivos multifuncionais passaram a ser categorizados de acordo com os equipamentos do SEP a serem protegidos. 


\subsection{Relés de proteção}

Relés de proteção são dispositivos configuráveis capazes de monitorar seções de um sistema elétrico. As grandezas enviadas ao relé são usualmente elétricas, mas podem ser de natureza mecânica, térmica, ou ainda uma combinação dessas.

$\mathrm{Na}$ corrida tecnológica para o desenvolvimento dos relés digitais, cada fabricante adotou uma solução proprietária em função da ausência de padronização, prejudicando a interoperabilidade entre relés de diferentes marcas. Nesse sentido, esforços foram feitos para trazer a possibilidade de integração entre fabricantes (HAN et al., 2014). A norma IEC 61850 (IEC, 2013) definiu diretrizes e protocolos que padronizaram a comunicação e operação de relés de diversos fabricantes. Desta forma, as grandezas, anteriormente citadas, passaram a ser digitalizadas e transmitidas, seguindo os protocolos SV e/ou GOOSE (OLIVEIRA; LOPES, 2018; ALI et al., 2018). A escolha das grandezas monitoradas está relacionada com as situações anormais que se deseja detectar durante o funcionamento do equipamento protegido.

\subsection{Dispositivos Eletrônicos Inteligentes (IEDs)}

De acordo com Cheng et al. (2017), os IEDs são sistemas computacionais programáveis, com hardware robusto para o ambiente industrial, dedicados a serviços úteis à automação de processos. Nos SEP, os relés digitais se enquadram na categoria de IED, executando diversas funcionalidades na automação destes sistemas. Ainda, segundo o autor supracitado, estes dispositivos realizam a medição de grandezas físicas provenientes de sensores; executam algoritmos de controle e proteção; atuam em equipamentos de manobra; armazenam o registro histórico do estado do SEP (através de listas de eventos e/ou oscilografias); e realizam comunicação com sistemas Supervisórios de Controle e Aquisição de Dados (SCADA) para operação em tempo real.

As interfaces de comunicação dos relés digitais também são utilizadas para a transferência de informação entre o relé e outros IEDs. Nos Sistemas de Automação de Subestação (SAS) atualmente instalados, são utilizadas interfaces dedicadas para o envio de sinais de teleproteção entre relés situados em diferentes subestações. Com o esforço 
unificador presente na norma IEC 61850, a interface Ethernet se tornou o meio preferencial para comunicação com IEDs em SAS.

\section{PROTEÇÃO USANDO TELECOMUNICAÇÕES}

O desenvolvimento de novas tecnologias aplicadas às telecomunicações facilitou a interconexão entre os sistemas de proteção, permitindo a integração dos relés responsáveis pela proteção de equipamentos do SEP, tais como barramentos de SEs, reatores e geradores. Os trabalhos de Cigré (2001), Ferrer e Scheitzer (2010) trazem informações detalhadas acerca das principais alternativas de equipamentos e tecnologias disponíveis para a proteção dos SEP. No Brasil, os requisitos técnicos dos sistemas de teleproteção são estabelecidos pelo Operador Nacional do Sistema (ONS), especificamente, na seção 6.2.1.9 do documento (ONS, 2011b). De acordo com os mesmos autores supramencionados, podese classificar os esquemas de proteção aplicados a LTs como:

\subsection{Esquemas de comparação analógicos}

Esses arranjos são baseados na comparação de grandezas analógicas associadas à LT. Com o aumento da capacidade de transmissão dos sistemas de telecomunicação, os sistemas de teleproteção, que antes se limitavam a enviar mensagens contendo apenas a direcionalidade e/ou do ângulo de fase da corrente, passaram a transmitir pacotes contendo diversos parâmetros de diferentes grandezas, conforme a constatação (FERRER; SCHEITZER, 2010).

Considerando que a adequada atuação da proteção depende de medições realizadas na mesma base de tempo, tornou-se necessária a implementação de técnicas de sincronização das mensagens. Inicialmente, as leituras dos equipamentos eram programadas para ocorrerem dentro de uma dilatada janela de tempo, como forma de compensação dos diferentes atrasos de transmissão. De acordo com Ferrer e Scheitzer (2010), atualmente, essa questão está plenamente resolvida com a adoção de equipamentos GPS, os quais possuem uma referência de tempo global e bastante precisa, permitindo que as mensagens sejam rotuladas com a base de tempo de suas respectivas medições. 
Conforme constatação de Pinto (2014), apesar dos esquemas analógicos serem aplicáveis a qualquer tipo de LT e nível de tensão, estes são particularmente vantajosos para LT's pequenas de até $80 \mathrm{~km}$ de extensão; linhas paralelas de múltiplos terminais; ou na ausência de transformadores de potencial (TPs) nos terminais da linha.

Mamede Filho e Mamede (2011) ressaltam que a teleproteção só é necessária para a proteção diferencial em LT, pois a distância entre os dois terminais torna indispensável o uso de telecomunicações para troca de informações entre eles. Para os demais esquemas de proteção, um único relé é suficiente, pois basta o mesmo receber, localmente, as leituras dos transformadores de corrente (TCS).

De acordo com Cigré (2001), outra particularidade desses métodos é que o comando de abertura de disjuntor é sempre instantâneo. A princípio, nos casos em que a seleção de fases é necessária para comandos de abertura monopolares, utiliza a técnica segregação de fases, ou seja, uma atuação distinta para cada fase do circuito. Segundo Ferrer e Scheitzer (2010), atualmente, com a adoção de sistemas de telecomunicação mais modernos e o uso de canais de comunicação mais confiáveis, são bastante comuns que os comandos para todas as fases sejam enviados por uma única via.

Os três tipos de comparação analógica mais utilizados são: proteção diferencial por corrente, conforme ilustra a Figura 2, proteção diferencial por comparação de fase e esquema de comparação de carga.

Figura 2 - Esquema representando a proteção diferencial analógica.

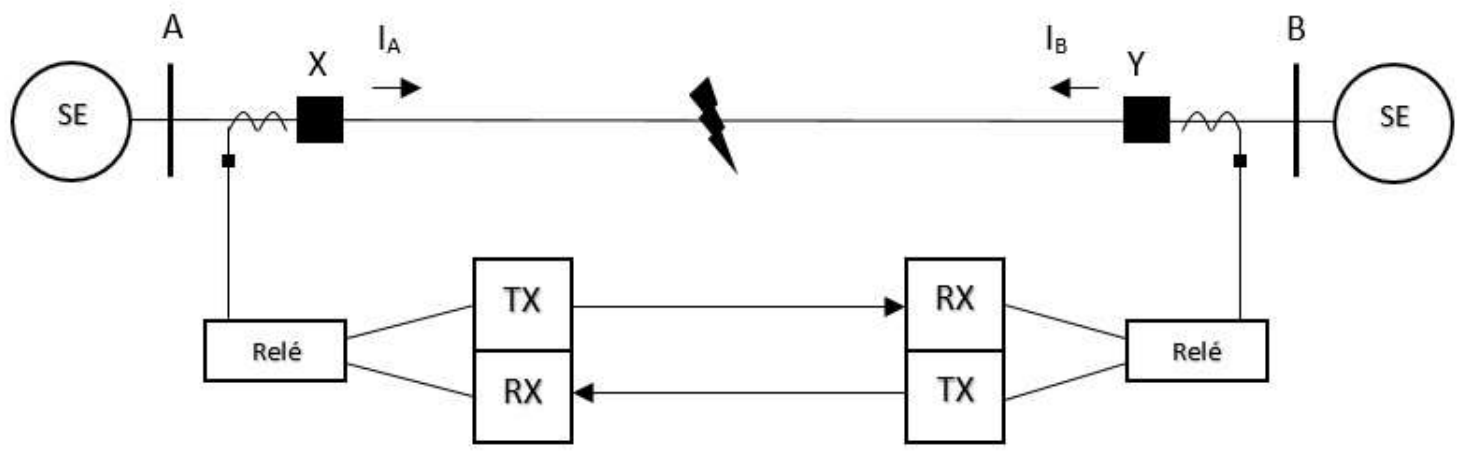

Fonte: Ferrer e Scheitzer (2010). 


\subsection{Esquemas de comparação de estado}

Esquemas de comparação de estado utilizam canais de comunicação para compartilhar informações lógicas de estados em ambos os terminais da linha. Segundo Cigré (2001), as informações que trafegam por esses canais de comunicação são: direcionalidade da corrente; status do disjuntor; bloqueio do religamento automático; transferência de disparo e outros. No Brasil, o ONS recomenda, na seção 8.2.3 do documento (ONS, 2011b), que os esquemas de transferência de disparo devam ser redundantes e independentes entre si.

De acordo com Mamede Filho e Mamede (2011), a comparação da direcionalidade da corrente, em ambos os terminais da linha, permite que se identifique se a origem do defeito é interna ou externa à linha. Relés de distância com direcionalidade ou de sobrecorrente direcionais, são utilizados em ambos os terminais. Dependendo da origem da falta, um relé pode comunicar-se com outro para que este acelere sua atuação, caso a falta seja interna à $\mathrm{LT}$, ou bloqueie o disparo de abertura do disjuntor do terminal remoto, caso a falta seja externa. É importante considerar que, mesmo com o bloqueio, o relé ainda poderá atuar para faltas externas caso isso seja previamente configurado. Os esquemas de comparação de estados mais conhecidos e aplicados são:

\subsubsection{Esquema de Transferência de Disparo Direto por Subalcance - DUTT}

A Figura 3 apresenta um exemplo desta aplicação. O esquema está suscetível a falhas, uma vez que ruídos e informações incorretas podem causar a abertura indesejada da LT. Para mitigar atuações indevidas na transferência de disparo, são utilizadas soluções que incluem verificação da integridade do dado ou o uso de dois canais separados para envio da mesma informação. 
Figura 3 - Esquema de Transferência de Disparo Direto por Subalcance.
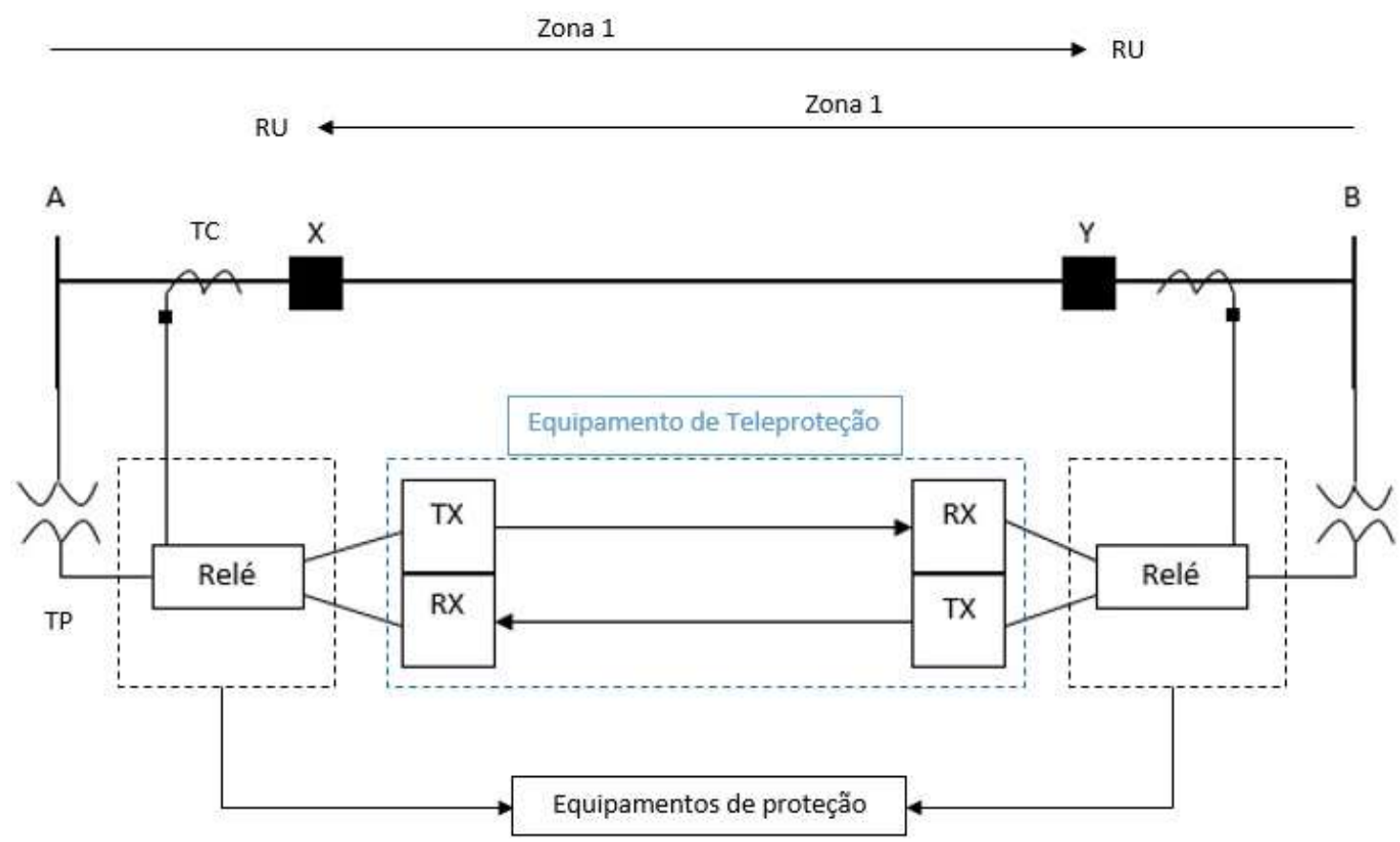

Fonte: Autor (2020).

Para a Figura 3, tem-se que RU: Função de subalcance; Zona 1: Zona de subalcance ou primeira zona de proteção da linha; A e B: Terminais da linha; X e Y: Disjuntores dos terminais A e B, respectivamente; TC e TP: Transformadores de corrente e de potencial, respectivamente; TX/RX: Transmissão e recepção, respectivamente. 
5.2.2 Esquema de Transferência de Disparo Permissivo por Subalcance - PUTT

Figura 4 - Esquema de Transferência de Disparo Permissivo de Subalcance.
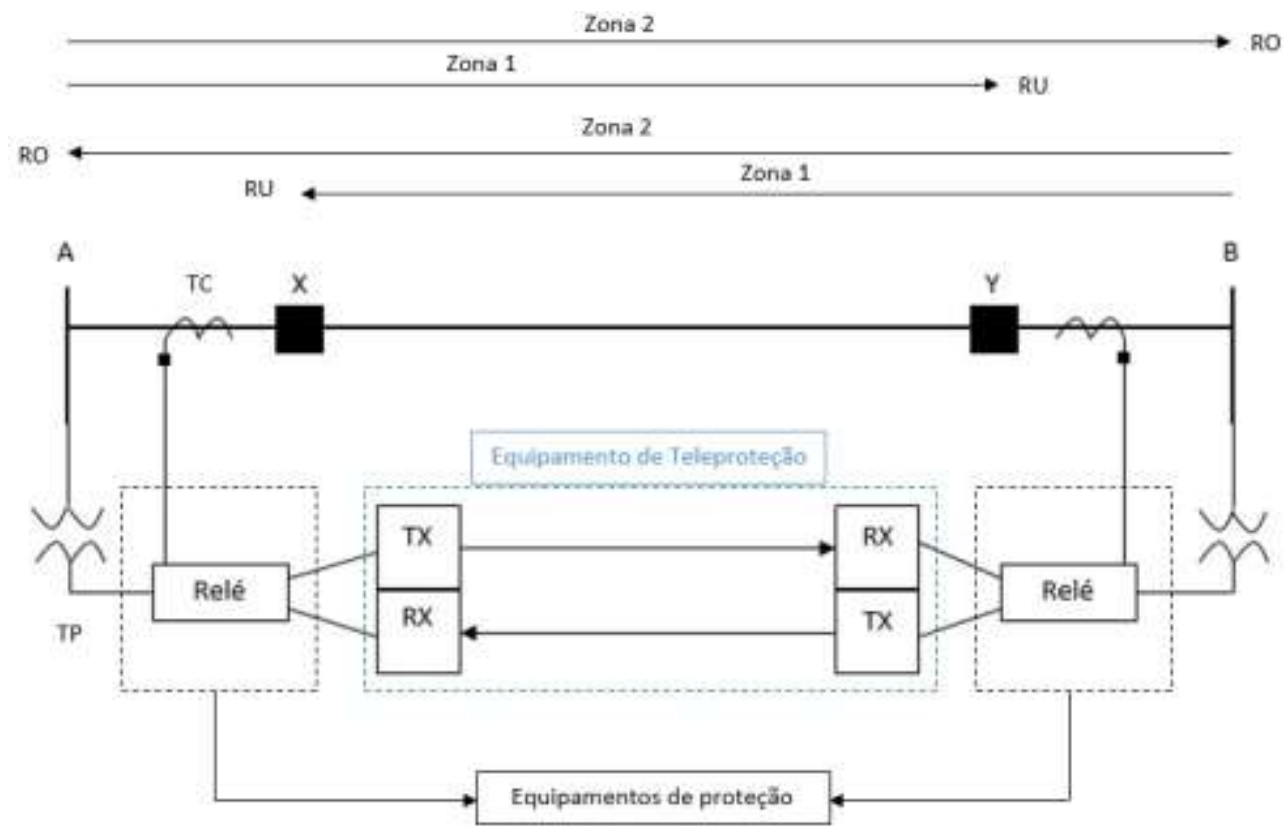

Fonte: Autor (2020).

Em condições normais, um sinal guarda Ferrer e Scheitzer (2010) é enviado constantemente através do canal de comunicação. Quando a função de subalcance (RU) detecta uma falta, é efetuado um disparo para abertura do disjuntor local e é enviada uma permissão de abertura para a unidade remota. Esse relé remoto, ao detectar a falta, terá a confirmação pelo outro terminal de que deve efetuar o comando de abertura ao seu disjuntor. Um exemplo desta aplicação pode ser observado na Figura 4.

O uso desse esquema não é permitido no Brasil, segundo o que é estabelecido na seção 6.2.1.9 do documento (ONS, 2011b). A justificativa é que para condições de weak infeed (WANG; CHEN, 2011), seja por uma fonte pequena em um dos terminais ou por uma falta de alta impedância, tal defeito só será eliminado a partir da temporização da zona de sobrealcance (zona 2), o que contraria a seção 6.2.1.11 do mesmo documento, com relação ao tempo máximo de eliminação de uma falta, já que o retardo inerente à atuação na referida zona não permite $o$ atendimento ao requisito temporal.

Para a Figura 4, tem-se que RO: Função de sobrealcance; Zona 2: Zona de sobrealcance ou segunda zona de proteção da linha. 
5.2.3 Esquema de Transferência de Disparo Permissivo por Sobrealcance - POTT

Figura 5 - Esquema de Transferência de Disparo Direto por Sobrealcance.
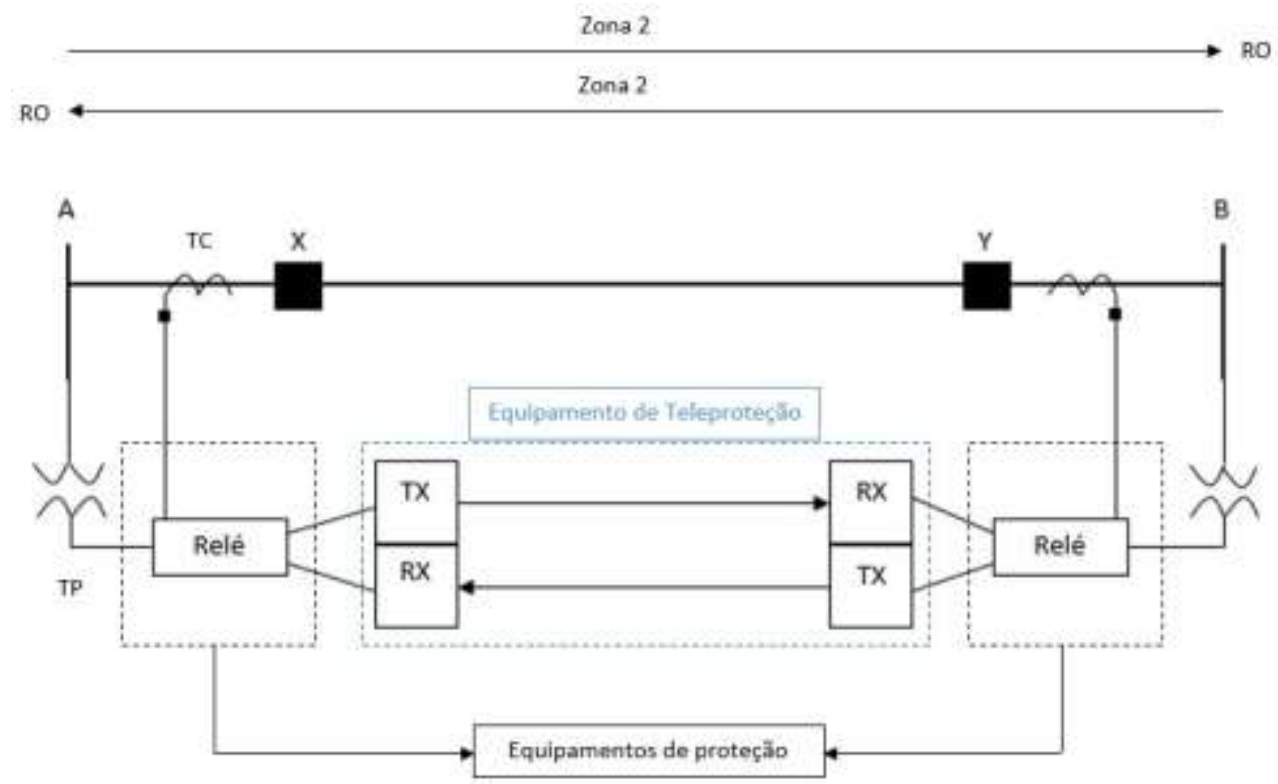

Fonte: Autor (2020).

Ao contrário do PUTT, esse arranjo só utiliza funções de sobrealcance. A Figura 5 auxilia o entendimento: um relé localizado em um dos terminais irá detectar a falta com sua função de sobrealcance e transmitir a permissão de disparo para o outro terminal. O outro relé, que receber o sinal, ao detectar a falta, abrirá o seu disjuntor e devolverá o sinal de abertura para o outro terminal operar. De acordo com Ferrer e Scheitzer (2010), a devolução do sinal para o relé do terminal inicial é chamada de lógica de Echo.

\subsubsection{Esquema de Prolongamento ou Aceleração da Zona}

Neste esquema as zonas se sobrepõem no meio da LT para evitar que faltas nessa região não sejam vistas por nenhum dos relés. Um exemplo disso pode ser visto na Figura 6. 
Figura 6 - Esquema de Prolongamento ou Aceleração de Zona.

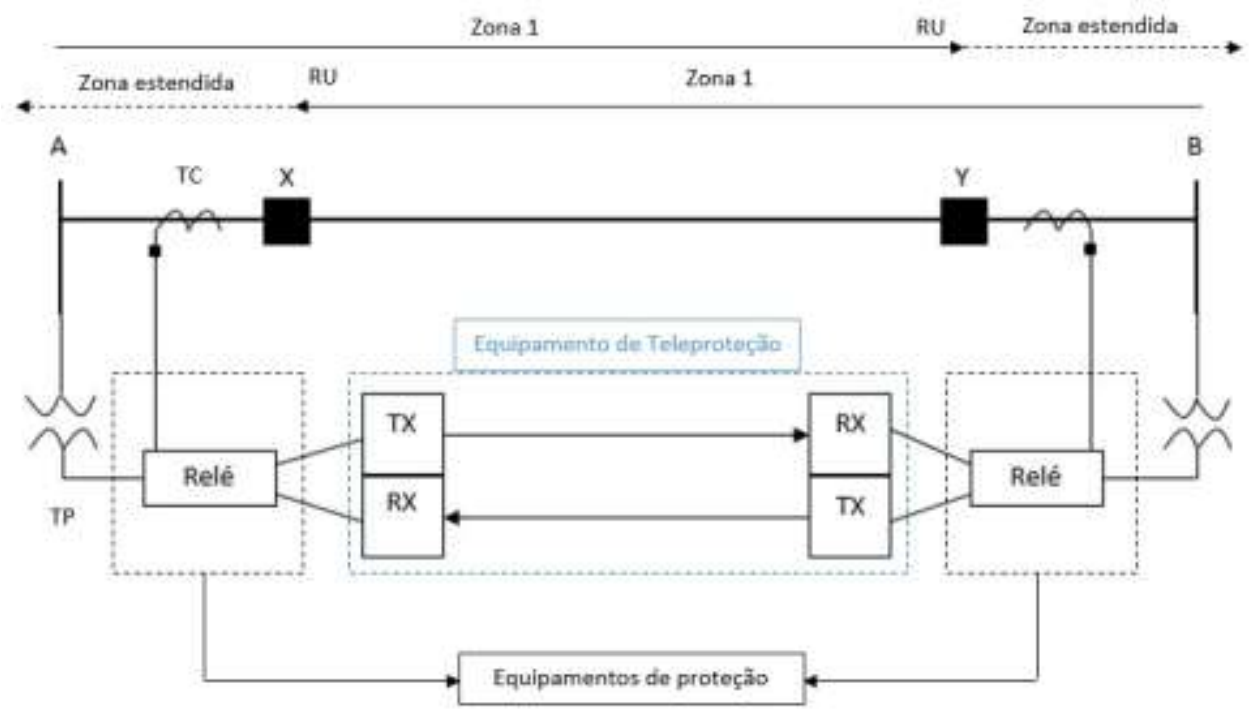

Fonte: Autor (2020).

5.2.5 Esquema de Comparação Direcional por Sinal de Bloqueio - DCB

Como mostrado na Figura 7, à atuação desse arranjo consiste em uma função de sobrealcance e outra de bloqueio. Sendo a de bloqueio responsável por impedir que a proteção da linha atue para faltas externas a ela.

Figura 7 - Esquema de Comparação Direcional por Sinal de Bloqueio.
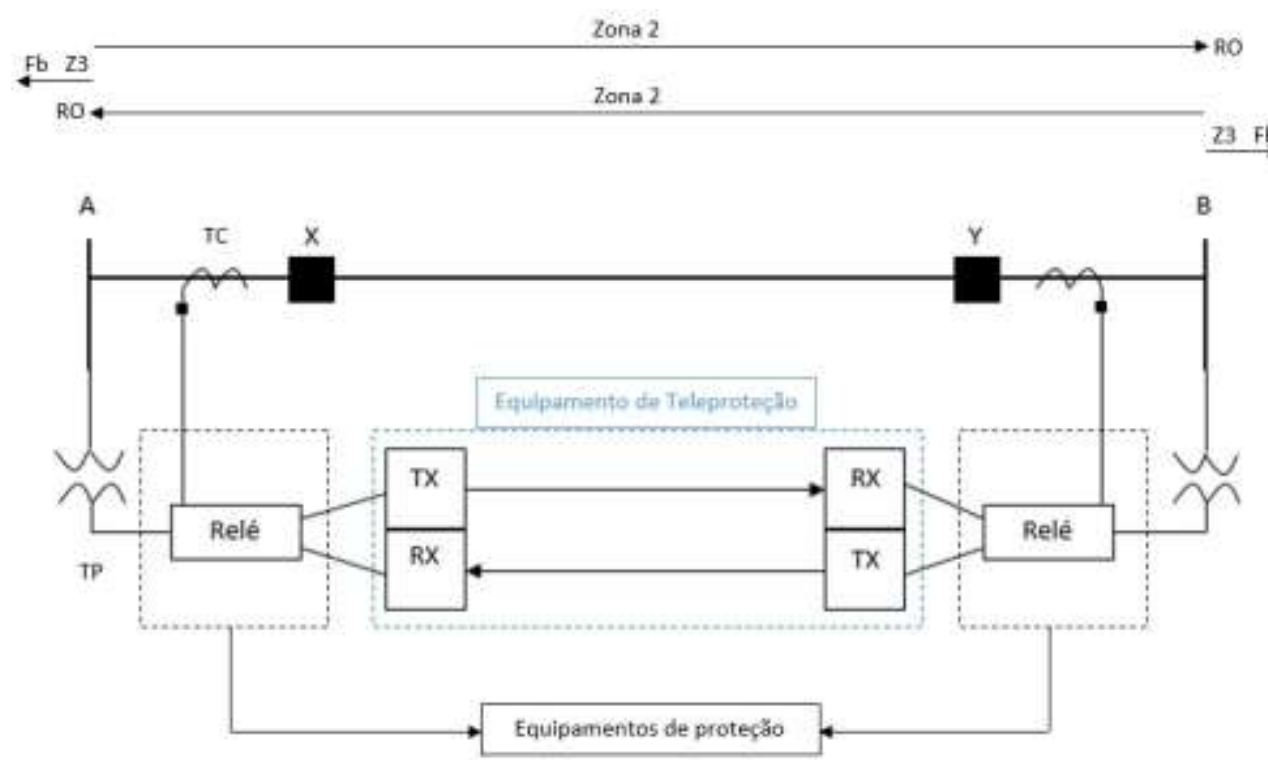

Fonte: Autor (2020). 
Para a Figura 7 tem-se que Fb: Função de bloqueio; Z3: Zona 3 ou terceira zona de proteção. Nesse caso esta aparece como uma zona reversa (MAMEDE FILHO; MAMEDE, 2011), mas poderia ser considerada uma zona de sobrealcance, em relação à Zona 2.

\subsubsection{Esquema de Comparação Direcional por Sinal de Desbloqueio - DCUB}

Este esquema funciona de forma similar ao esquema POTT. Um sinal de guarda é continuamente enviado entre os terminais da LT e quando a função de sobrealcance de um dos terminais detecta uma falta, é enviado um sinal de atuação para o outro terminal. O relé do outro terminal, ao perceber a mudança do sinal de guarda para um sinal de atuação, irá verificar se a sua função de sobrealcance também detectou a falta. Caso ele também detecte, abrirá o disjuntor e devolverá o sinal de abertura para que o relé do terminal inicial possa abrir também, ou seja, a função de proteção de um dos terminais irá desbloquear a atuação da função do outro terminal. (FERRER; SCHEITZER, 2010).

Uma inovação desse método é que ele possui uma lógica de atuação que engloba casos em que o canal de comunicação esteja fora de operação. Ao verificar que o canal está fora de operação, à detecção da falta pela função de sobrealcance será o suficiente para a abertura do disjuntor, não sendo necessário que outro terminal a desbloqueie (CIGRÉ, 2001). Na Figura 8 é ilustrado o esquema.

Figura 8 - Esquema de Comparação Direcional por Sinal de Desbloqueio.

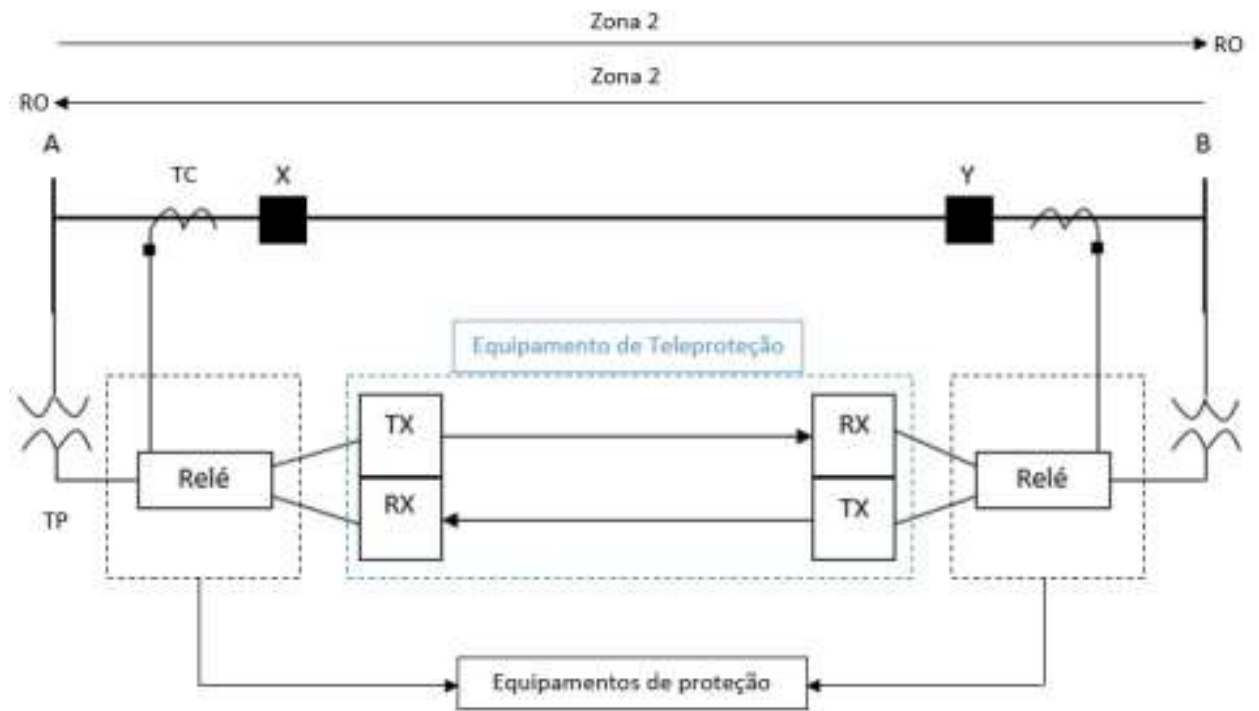

Fonte: Autor (2020). 


\section{SISTEMAS DE COMUNICAÇÃO APLICADOS À PROTEÇÃO}

Disponibilidade, confiabilidade e simplicidade são as principais métricas para comunicações de teleproteção (DOLEZILEK et al., 2015). A implantação de redes digitais foi motivada, inicialmente, pelo o aumento do tráfego de dados proveniente da contínua inserção de novos subsistemas consumidores, unidades de geração distribuída e pela consequente necessidade de aumento da capacidade de controle da rede. (CIGRÉ, 2001).

$\mathrm{O}$ atendimento às demandas foi mais rápido que as iniciativas de padronização dos respectivos sistemas de comunicação. Desta forma, atualmente, existe uma tendência de se combinar todos os padrões em uma rede Ethernet. Essa solução parece viável econômica e tecnicamente, no entanto, esbarra em algumas ressalvas: os sinais de proteção trafegam em um meio de comunicação de largura de banda compartilhada, e estão sujeitos a uma rede que não foi projetada para tempos de entrega determinísticos (DOLEZILEK et al., 2015) .

O uso de uma rede Ethernet para sinais de teleproteção enfrenta ainda outros desafios. O ONS define três requisitos fundamentais para a teleproteção. A seção 8.2 do documento (ONS, 2011b) recomenda que os canais para teleproteção devam ser dedicados especificamente para proteção e não compartilhados com outras aplicações. Os esquemas de teleproteção devem ser independentes e redundantes para a proteção principal e alternada, utilizando meios físicos de transmissão independentes, de tal forma que a indisponibilidade de uma via de telecomunicações não comprometa a disponibilidade da outra via. Ademais, os esquemas de transferência de disparo devem ser independentes e redundantes para a proteção principal e alternada. Para a utilização de uma Rede definida por Software (SDN) na teleproteção, estes requisitos precisam ser revistos para a inclusão de novos parâmetros e critérios.

Diante deste cenário, pode-se afirmar que o projeto adequado da rede de telecomunicações é um pré-requisito fundamental para a proteção de sistemas elétricos desdobrados em grandes áreas (CIGRÉ, 2001). As principais questões a serem consideradas para planejamento e implantação são: 


\subsection{Interfaces de comunicação dos IEDs para teleproteção}

Em Cigré (2001), as interfaces são classificadas segundo os seguintes critérios: localização do ponto de conexão e meio físico de transmissão. Quanto ao critério de localização, dois tipos de interfaces são definidos. O tipo (a) corresponde à interface para comunicação entre o dispositivo de teleproteção e o relé de proteção, isto é, o dispositivo capaz de realizar a comunicação e o atuador. Já a interface do tipo (b) permite a conexão entre o dispositivo de teleproteção e o sistema de telecomunicação.

Com relação aos meios físicos de transmissão, encontram-se três classes de interfaces: de contato, analógicas e digitais.

Destacando-se as interfaces digitais, conforme Cigré (2001), estas são categorizadas como seriais, óticas e Ethernet. As normas associadas às interfaces digitais de fibra ótica definem padrões para conectores, interfaces para LANs óticas e sistemas de telecomunicação. A fibra ótica permite grande flexibilidade de conexão e é atualmente a interface digital mais utilizada.

A interface digital Ethernet/LANs teve aplicação, a priori, para comunicação do controle local da subestação (CIGRÉ, 2001). Com a padronização da norma IEC 61850, o uso dessa interface foi ampliado para novas funções correspondentes a controle e proteção.

Como exemplo de interfaces de relés de proteção digital com tecnologia fibra ponto a ponto, citam-se os relés ABB RED670 e REL670 com IEEE C37.94 ou G.703 (HITACHI ABB, 2020).

\subsection{Tecnologias de telecomunicações disponíveis}

Os equipamentos de proteção precisam se adaptar continuamente às novas tecnologias, no entanto, devido à criticidade desses dispositivos, associada a longos períodos de desenvolvimento, as tecnologias de comunicação para teleproteção estão sempre um ou dois passos à frente, como observado na Figura 9. 
Figura 9 - Ciclos de vida das tecnologias de comunicação aplicadas à teleproteção.

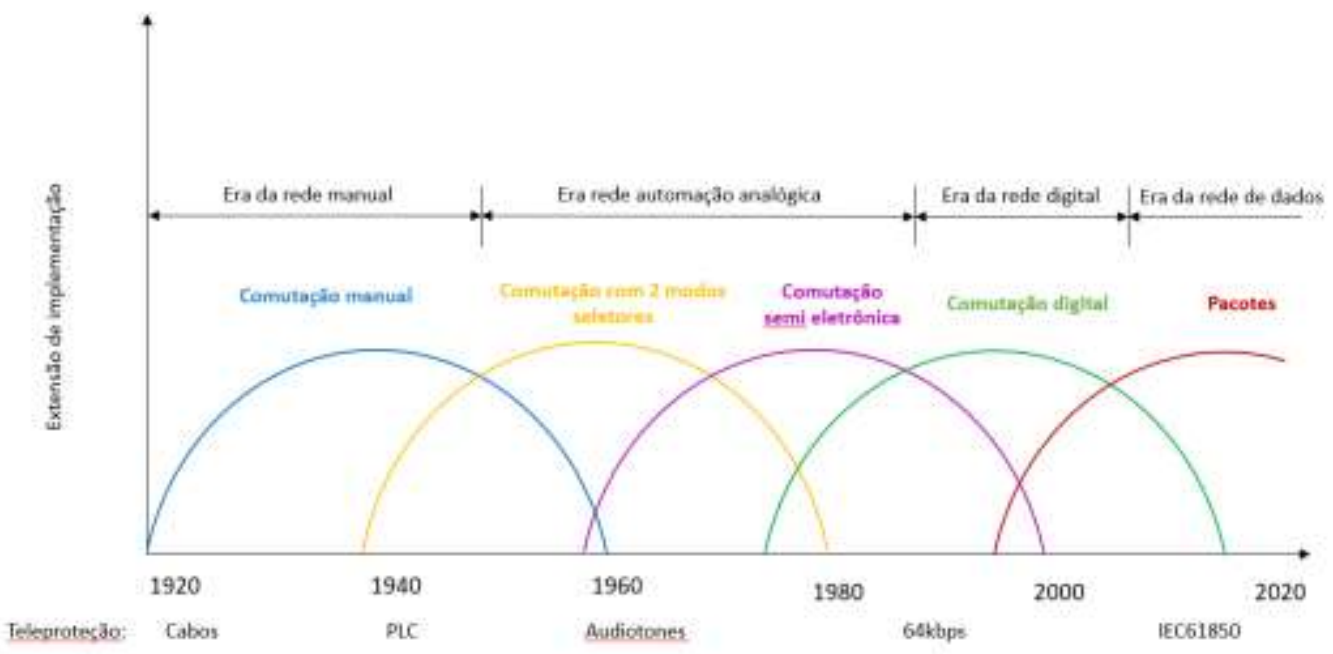

Fonte: Rahman et al. (2018).

A escolha do meio de transmissão para teleproteção está associada a duas condicionantes principais: o legado de comunicação já instalado na área do SEP e os requisitos técnicos dos esquemas de proteção.

As linhas de alta tensão passaram a figurar também como meio de transmissão com o surgimento da tecnologia denominada Power Line Carrier (PLC). Os sistemas PLC foram intensamente utilizados em linhas de alta tensão para a transmissão de voz, dados de controle e sinais de proteção (CIGRÉ, 2001). Tais sistemas representam os enlaces mais curtos e de conexão direta com os extremos da linha, estações ou subestações de energia, justificando a sua utilização para transmissão de sinais de proteção. Apesar de ser um sistema com alta redundância (várias LTs), de ser um meio de transmissão já instalado e proprietário da concessionária, ele vem caindo em desuso para a proteção primária, em função das seguintes constatações: faltas na linha geram transientes com forte ruído no canal PLC o que reduz capacidade de transmissão e aumenta latência do sistema; não se aplica a proteção diferencial e a banda limitada do canal de transmissão restringe o número de sinais de proteção a serem transmitidos.

De acordo com Cigré (2001), os canais de ondas de rádio atenderam a uma crescente demanda por capacidade de comunicação de diversas concessionárias. Segundo o IEEE (2013), antes da adoção da fibra ótica para aplicação em sistemas de proteção, os enlaces de micro-ondas representavam o único meio de transmissão em banda larga. Outro 
motivo para utilização desta tecnologia é a considerável imunidade à interferência eletromagnética produzida pelas redes de alta tensão.

De acordo com a última referência citada, os principais obstáculos que impedem o uso intensivo desta tecnologia são: capacidade de transmissão influenciada por condições atmosféricas; necessidade de linha com visada livre entre as antenas dos transceptores; em distâncias maiores que $50 \mathrm{~km}$ necessidade de abordagem múltiplos saltos, o que eleva o custo e aumenta a latência do sistema.

A fibra ótica vem se desenvolvendo desde a década de 80 , sendo considerada, atualmente, a melhor opção para a transmissão de dados em banda larga, cobrindo grandes distâncias. Para maximizar o uso da banda disponível da fibra, foram adotadas técnicas de multiplexação, em especial o TDM, para organização do tráfego em diferentes hierarquias digitais. Inicialmente, as iniciativas para oferecer equipamentos com essas características não tiveram um padrão a ser seguido, o que, na prática, inviabilizou a integração de redes com equipamentos de diferentes fabricantes.

Criado nos Estados Unidos, o SONET foi o primeiro padrão de hierarquia digital a ser amplamente utilizado. Em seguida, a ITU-T (Europa) fez algumas recomendações (G.707, G.708 e G.709) ao padrão americano, que culminaram no estabelecimento do padrão SDH.

Apesar de terem surgido para utilização em fibras óticas, as tecnologias SONET/SDH podem ser usadas em outros meios de transmissão, tais como enlaces de rádios digitais.

\subsubsection{Principais tecnologias de rede aplicadas à teleproteção}

Uma rede de telecomunicação é um conjunto de equipamentos de comunicação e comutação, atuando de forma colaborativa para prover serviços de telecomunicações em pontos de acesso distribuídos em uma determinada área (CIGRÉ, 2001).

- $\quad$ Switched Networks (ATM)

Segundo Siu e Jain (1995), o ATM é capaz de suportar todas as classes de tráfego (voz, vídeo, dados), além de permitir à implementação de serviços que proporcionem acesso mais flexível a rede utilizada.

Os autores em Ali et al. (2018) fizeram a validação de esquemas de proteção diferencial de linha, utilizando o padrão da IEC 61850 sobre ATM e concluíram sobre a perfeita aderência aos requisitos de temporização para aplicações de teleproteção. 
Assim, o ATM pode ser considerado uma solução viável para a teleproteção, desde que os requisitos gerais de Qualidade de Serviço (QoS) estejam sob o controle do administrador do sistema de proteção.

- $\quad$ Multi-Protocol Label Switching (MPLS)

Atualmente, a tecnologia de roteamento de pacotes mais difundida para Redes de Comunicação Extensas (WANs) é o MPLS. Esta tecnologia é definida pela RFC 3031 e está situada entre as camadas 2 e 3 do modelo OSI. Em relação às redes tradicionais (Intenet Protocol), o MPLS se mostra muito superior, pois tem a capacidade de otimizar o tráfego da rede, de modo a se adaptar às diferentes demandas.

O trabalho publicado em Rahman et al. (2018) fez uma avaliação da aplicação do MPLS em teleproteção, chegando às seguintes conclusões: as redes MPLS são viáveis quando projetadas considerando latência, assimetria, tempo de recuperação de falhas e disponibilidade; esta tecnologia aumenta a capacidade de monitoramento e diagnóstico de falhas, permitindo a localização das mesmas dentro da cadeia de comunicação.

\subsubsection{Requisitos gerais dos sistemas de telecomunicações}

O sistema de telecomunicação deve atender aos requisitos do sistema de proteção. Para a parametrização do sistema de telecomunicações utilizam-se as seguintes terminologias: tempo de propagação, simetria do tempo de propagação, variação do tempo de propagação (Jitter), tempo de transmissão, tempo de operação da teleproteção e tempo de operação de proteção. O relatório de Cigré (2001) define os tempos de referência conforme a Figura 10.

A norma IEEE 1646 IEEE (2005) e Fodero, Huntley e Robertson (2018) apresentam os requisitos temporais para comunicação em um SAS. Os principais requisitos temporais e os tempos máximos de transferência apontados em IEEE (2005) são: transferência de informação de proteção entre subestações (8 a 12 ms); Disparo (Trip) de disjuntor e partida de falha de disjuntor (4 ms); disparo (Trip) de disjuntor reserva (depois de tempo expirado de falha de disjuntor) (8 a $12 \mathrm{~ms}$ ); religamento de disjuntor (incluindo religamentos com supervisão de tensão e múltiplos) (8 ms); Controle de transferência de disparo para comandos de ENVIO-RECEBIMENTO (4 ms); Chaveamento para esquemas permissivos (8 
ms); ENVIO-RECEBIMENTO de comandos de disparo (Trip) (2 a 8 ms) e Partida de função de bloqueio (não em bloqueio mecânico) (16 ms).

Figura 10 - Requisitos temporais de teleproteção.

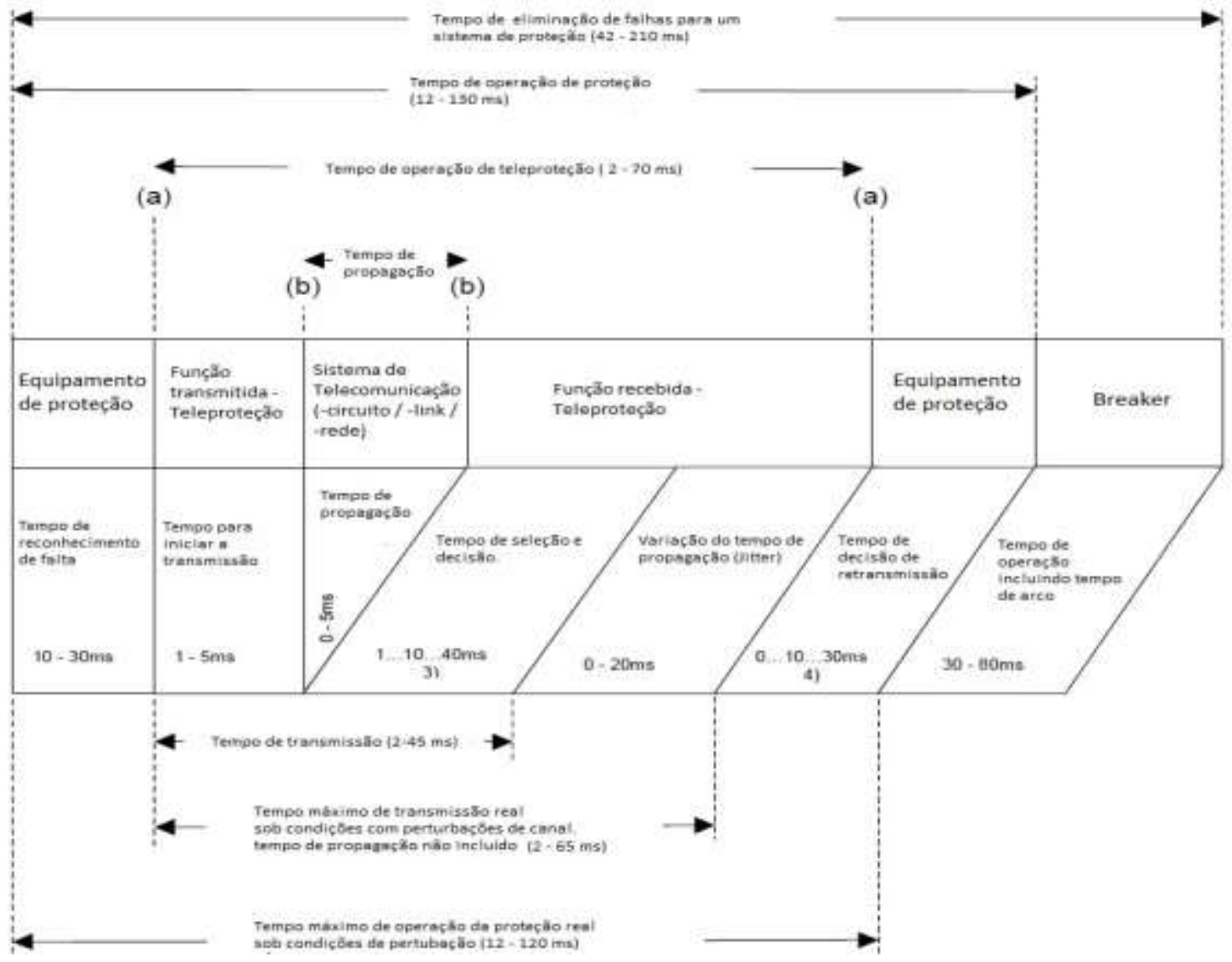

Fonte: Cigré (2001).

As seções 6 e 7 do documento ONS (2011b) definem que o tempo mínimo para registrar cada perturbação é de 160 ms de dados de pré-falta e possuir um tempo pós-falta ajustável entre 100 e 5000 ms. O tempo de atraso entre a atuação de qualquer canal digital e o registro do respectivo sinal não pode exceder 2 ms e o tempo para eliminação de faltas, incluindo a abertura de todos os disjuntores da LT, não deve exceder 100 ms.

\section{CONCLUSÃO}

Este artigo apresenta os conceitos gerais de proteção dos SEP, os esquemas de teleproteção e as respectivas tecnologias de telecomunicação associadas. Adicionalmente, foram apresentados parâmetros técnicos de referência e citados diversos trabalhos de 
pesquisa do estado da arte, referente ao assunto em tela. Diante da consolidação de todas essas informações, este texto se traduz em uma destacada referência para profissionais e pesquisadores que desejam se aprofundar nos conceitos e tendências tecnológicas relacionados à teleproteção.

Por fim, cabe dar destaque ao trabalho de Dolezilek et al. (2015) que aponta a SDN como a tecnologia destinada a revolucionar a forma de se projetar, operar e manter redes críticas, permitindo aos engenheiros de rede a capacidade de abstração dos sistemas de comunicações de teleproteção no cenário Ethernet, tratando os circuitos virtuais dedicados, sem sacrificar a simplicidade, flexibilidade e confiabilidade.

\section{AGRADECIMENTOS}

Os autores agradecem a empresa Transmissora de Energia Elétrica S.A. - TAESA, pelo apoio financeiro durante o desenvolvimento deste artigo no projeto de $P \& D$ com código ANEEL PD-07130-0053/2018.

\section{REFERÊNCIAS}

ALI, I. et al. Communication modeling for differential protection in IEC-61850-based substations. IEEE Transactions on Industry Applications, [S. I.], v. 54, n. 1, p. 135-142, jan./fev. 2018. Disponível em: https://ieeexplore.ieee.org/document/8010855. Acesso em: 12 fev. 2020.

ARAGÃO, B. L. C. S. et al. Sistema de comunicação utilizando cabos OPGW para monitoramento de linhas de transmissão. In: ENCONTRO ANUAL DO IECOM EM COMUNICAÇÕES, REDES E CRIPTOGRAFIA, 2016, Fortaleza. Anais [...]. Fortaleza: ENCOM, 2016. p. 1-2.

CHENG, X. et al. Modernizing substation automation systems: adopting IEC standard 61850 for modeling and communication. IEEE Industry Applications Magazine, v. 23, n. 1, p. 42-49, jan./fev. 2017. Disponível em: https://ieeexplore.ieee.org/document/7725502. Acesso em: 12 fev. 2020.

CIGRÉ. Protection using telecommunications. [Paris]: CIGRÉ, 2001. Disponível em: https://ecigre.org/publication/192-protection-using-telecommunications. Acesso em: 12 fev. 2020.

DECHUN, W.; ZHIHUI, F.; JIE, T. The study of temperature measurement on optical fiber in OPGW based on stimulated brillouin scattering. In: 2016 IEEE INTERNATIONAL CONFERENCE ON INFORMATION AND AUTOMATION (ICIA), 2016, Ningbo. Anais [...]. Ningbo: IEEE, 2016, p. 17531757. Disponível em: https://ieeexplore.ieee.org/document/7832101. Acesso em: 12 fev. 2020.

DOLEZILEK, D. et al. Simplifying teleprotection communications with new packet transport technology. In: INTERNATIONAL SCIENTIFIC AND TECHNICAL CONFERENCE: ACTUAL TRENDS IN 
DEVELOPMENT OF POWER SYSTEM RELAY PROTECTION AND AUTOMATION, 5., Johannesburg. Anais [...].Johannesburg: Schweitzer Engineering Laboratories, 2015. p. 1-11. Disponível em: https://cms-

cdn.selinc.com/assets/Literature/Publications/Technical\%20Papers/6697_SimplifyingTeleprotect ion_DD_20150422_Web2.pdf. Acesso em: 12 fev. 2020.

FERRER, H. J. A.; SCHEITZER, E. O. Modern solutions for protection, control, and monitoring of electric power systems. Pulman: Schweitzer Engineering Laboratories, 2010.

FODERO, K., HUNTLEY, C., ROBERTSON, P. Deterministic communications for protection applications over packet-based wide-area networks. In: Annual Conference for Protective Relay Engineers (CPRE), 71., 2018, College Station. Anais [...]. College Station: IEEE, 2018, p. 1-6. Disponível em: https://ieeexplore.ieee.org/document/8349789. Acesso em: 12 fev. 2020.

FORTES, M. Z.; FERREIRA, V. H.; ZANGHI, R. Fault diagnosis in transmission lines: trends and main research areas. IEEE Latin America Transactions, [S. I.], v. 13, n. 10, p. 3324-3332, 2015. Disponível em: https://ieeexplore.ieee.org/document/7387238. Acesso em: 12 fev. 2020.

HAN, G. et al. An open communication architecture for distribution automation based on IEC 61850. International Journal of Electrical Power and Energy Systems, [S. I.], v. 54, p. 315-324, 2014. Disponível em: https://www.sciencedirect.com/science/article/abs/pii/S0142061513003104. Acesso em: 12 fev. 2020.

HITACHI ABB. The power of one solution for protection and control. HITACHI ABB, [S. I.], 2020. Disponível em: https://new.abb.com/substation-automation/products/protection-control. Acesso em: 12 fev. 2020.

IEC. IEC 61850 - Communication networks and systems in substations. [Genebra]: IEC, 2013.

IEEE. IEEE guide for power system protective relay applications over digital communication channels. IEEE Std C37.236-2013, [S. I.], p. 1-84, 2013. Disponível em: https://ieeexplore.ieee.org/document/6504869. Acesso em: 12 fev. 2020.

IEEE. IEEE standard communication delivery time performance requirements for electric power substation automation. IEEE Std 1646-2004, [S. I.], p. 1-36, 2005. Disponível em: https://ieeexplore.ieee.org/document/1405811. Acesso em: 12 fev. 2020.

IEEE. IEEE standard electrical power system device function numbers, acronyms, and contact designations. IEEE Std C37.2-2008, [S. I.], p. 1-48, 2008. Disponível em: https://ieeexplore.ieee.org/document/4639522. Acesso em: 12 fev. 2020.

KINDERMANN, G. Proteção de sistema elétricos de potência: volume I. Florianópolis: UFSC: EEL: LABPLAN, 2005.

LESSA, T. S. et al. Evaluation of carbon fiber cables in transmission lines. Electric Power Components and Systems, [S. I.], v. 46, n. 5, p. 544-559, 2016. Disponível em: https://www.tandfonline.com/doi/full/10.1080/15325008.2018.1455246. Acesso em: 12 fev. 2020. 
MAMEDE FILHO, J.; MAMEDE, D. R. Proteção de sistemas elétricos de potência. Rio de Janeiro: LTC, 2011.

MINISTÉRIO DE MINAS E ENERGIA. Boletim mensal de monitoramento do sistema elétrico brasileiro: junho / 2018. [Brasília, DF]: MME, 2018a. Disponível em: http://www.mme.gov.br/documents/239673/909080/Boletim+de+Monitoramento+do+Sistema +El\%C3\%A9trico+-+Junho+-+2018_rev1.pdf/62960eb1-778d-807e-341e-5ba8e3b67476. Acesso em: 12 fev. 2020.

OLIVEIRA, W.; LOPES, Y. Teleprotection over SONET based on IEC 61850. In: SIMPÓSIO BRASILEIRO DE SISTEMAS ELETRICOS, Niteroi. Anais [...].Niteroi: IEEE, 2018, p. 1-6. Disponível em: https://ieeexplore.ieee.org/document/8395834. Acesso em: 12 fev. 2020.

ONS. Submódulo 2.4: requisitos mínimos para linhas de transmissão. [Rio de Janeiro]: ONS, 2011a. Disponível em:

http://www.ons.org.br/\%2FProcedimentosDeRede\%2FM\%C3\%B3dulo\%202\%2FSubm\%C3\%B3du lo\%202.4\%2FSubm\%C3\%B3dulo\%202.4.pdf. Acesso em: 12 fev. 2020.

ONS. Submódulo 2.6: Requisitos mínimos para os sistemas de proteção e de telecomunicações. [Rio de Janeiro]: ONS, 2011b. Disponível em: http://www.ons.org.br/\%2FProcedimentosDeRede\%2FM\%C3\%B3dulo\%202\%2FSubm\%C3\%B3du lo\%202.6\%2FSubm\%C3\%B3dulo\%202.6_Rev_2.0.pdf. Acesso em: 12 fev. 2020.

PINTO, M. O. Energia elétrica: geração, transmissão e sistemas interligados. Rio de Janeiro: LTC, 2014.

RAHMAN, T. et al. Teleprotection with MPLS ethernet communications - development and testing of practical installations. In: Annual Conference for Protective Relay Engineers (CPRE), 71., 2018, College Station. Anais [...]. College Station: IEEE, 2018. p. 1-18. Disponível em: https://ieeexplore.ieee.org/document/8349828. Acesso em: 12 fev. 2020.

SANTOS, F. M. P.; BERNARDES, W. M. S.; ASADA, E. N. Coordenação de relés direcionais de sobrecorrente por meio de evolutionary particle swarm optimization. In: ENCONTRO REGIONAL IBERO-AMERICANO DO CIGRÉ, 15., 2013, Foz do Iguaçu. Anais [...]. Foz do Iguaçu: ERIAC, 2013, p. 1-8.

SIU, K.; JAIN, R. A brief overview of atm: protocol layers, lan emulation, and traffic management. ACM SIGCOMM Computer Communication Review, [S. I.], v. 25, n. 2, p. 6-28, 1995. Disponível em: http://arxiv.org/abs/cs/9809083. Acesso em: 12 fev. 2020.

WANG, M.; CHEN, Y. Weak infeed study and protection solution. In: WESTERN PROTECTION RELAY CONFERENCE, 2011, Spokane. Anais [...]. Spokane: WPRC, 2011, p. 1-12. Disponível em: https://na.eventscloud.com/file_uploads/136b969dbfc7734cdfd93780f709b85d_wan_pap.pdf. Acesso em: 12 fev. 2020. 